\title{
Sensor Based on the Mode-Localization Effect in Electrostatically-Coupled MEMS Resonators Fabricated Using an SOI Process
}

\author{
Graham S. Wood, Chun Zhao, \\ and Ibrahim Sari \\ Nano Research Group \\ School of Electronics and Computer Science \\ University of Southampton \\ Southampton, U.K. \\ gsw1g11@ecs.soton.ac.uk
}

\author{
Suan Hui $\mathrm{Pu}$ \\ University of Southampton Malaysia Campus \\ Nusajaya, Malaysia
}

\author{
Michael Kraft \\ Montefiore Institute \\ University of Liège \\ Liège, Belgium
}

\begin{abstract}
The mode-localization effect exhibited in electrostatically-coupled microelectromechanical systems (MEMS) resonators was demonstrated using a pair of rectangular clamped-clamped beams that were fabricated using a silicon-on-insulator (SOI) based process. The response of the amplitude ratio of the resonating beams at the fundamental mode frequencies to a change in the stiffness of one of the beams was characterized. Beams with different widths were fabricated and tested, with up to 13 times improvement in sensitivity to relative stiffness change being reported for a device with $20 \mu \mathrm{m}$ widths, compared to $10 \mu \mathrm{m}$ widths. In addition, when compared to the state-of-the-art, the devices reported up to 2.8 times improvement in sensitivity.
\end{abstract}

Keywords-Resonators; stiffness change; MEMS; modelocalization; sensor.

\section{INTRODUCTION}

There are many previously reported examples of microsensors exploiting the resonant frequency-shift of a MEMS resonator in order to measure stiffness [1] or mass change [2]. A more recent trend in MEMS-resonator based sensing is to apply DC voltages to two closely spaced resonators, creating an electric field between them that acts as a coupling spring. When an imbalance is introduced to the system, such as a mass or stiffness change to one of the resonators, a shift in the amplitude ratio of the resonant mode shapes occurs.

Previously reported research [3] has demonstrated that the response of the normalized mode shapes ("eigenstates") to a stiffness perturbation on one resonator in a coupled pair is three orders of magnitude greater than the response of the resonant frequency.

Building on a fabrication process reported previously [4], this paper will detail the mode-localization behavior of a pair of electrostatically-coupled rectangular clamped-clamped beams, differing from other work, which has characterized double-ended tuning forks [3] and wine glass ring resonators [5].

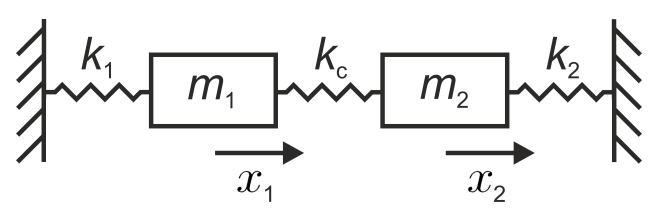

Fig. 1. Mass-spring model of a 2-DOF system consisting of two coupled resonators.

\section{THEORY}

A pair of electrostatically coupled MEMS resonators can be analyzed using the mass-spring model shown in Fig. 1, with each resonator consisting of a mass, $m$, and a spring, $k$. The electrostatic spring, which is formed by an electric field created between the resonators, is added to the model as $k_{c}$.

Starting from Hooke's Law and Newton's 2nd Law, theoretical analysis has shown that the coupled system of Fig. 1 possesses two fundamental mode frequencies, $f_{i p}$ and $f_{o p}$, where the resonators oscillate either in-phase or out-of-phase with respect to each other. An electrostatic spring has a negative spring constant, for which a minus sign accounts for, and the two frequencies are given by

$$
\begin{gathered}
f_{o p}=\frac{1}{2 \pi} \sqrt{\frac{k-2 k_{c}}{m}} \\
f_{i p}=\frac{1}{2 \pi} \sqrt{\frac{k}{m}}
\end{gathered}
$$

where $m_{1}=m_{2}=m$ and $k_{1}=k_{2}=k$ for a balanced system.

At each of the two modes, the response of the amplitude ratio $\left(x_{1} / x_{2}\right)$ to a stiffness perturbation is calculated. The stiffness of resonator 1 is increased by $\Delta k$, so that $k_{1}=k$ $+\Delta k$ and $k_{2}=k$, and the following expressions are obtained

$$
{\frac{x_{1}}{x_{2}}}_{o p}=\frac{-\frac{1}{2}\left(\Delta k-\sqrt{4 k_{c}^{2}+\Delta k^{2}}\right)}{-k_{c}}
$$




$$
{\frac{x_{1}}{x_{2} i p}}_{i p}=\frac{-\frac{1}{2}\left(\Delta k+\sqrt{4 k_{c}^{2}+\Delta k^{2}}\right)}{-k_{c}}
$$

It can been seen from (2) and (3) that the response of amplitude ratio to a stiffness perturbation is influenced by the coupling spring strength, $k_{c}$. It is found that the amplitude ratio is more sensitive to a given stiffness perturbation with a weaker coupling spring. However, from (1), it is noted that a weaker value for $k_{c}$ will cause the two mode frequencies to converge. Therefore, an important practical consideration is to choose a value for the coupling spring that is strong enough to avoid mode-aliasing, which would prevent accurate measurement of the amplitudes of the two resonators. The electrostatic coupling spring is achieved by DC biasing the two resonators to create a potential difference, $\Delta V$, between them, with the spring constant given by

$$
k_{c}=\frac{-(\Delta V)^{2} \varepsilon_{0} A}{g^{3}}
$$

where $g$ is the coupling gap between the resonators and $A$ is the cross section of the resonators at the coupling gap.

From (2) and (3), it is noted that the amplitude ratio response is not influenced by the initial balanced stiffness, $k$, of the resonators, so for a given $\Delta k$, the amplitude ratio response will be the same regardless of the initial $k$. Therefore, it is expected that the sensitivity of the amplitude ratio to a relative change in stiffness $(\Delta k / k)$ will be increased for devices with higher initial balanced stiffness, $k$.

\section{Design of Devices}

For this work, a coupled resonator device was fabricated using a dicing-free fabrication process [4], as shown in Fig. 2. Starting with an SOI wafer with a device layer of $50 \mu \mathrm{m}$ thickness and buried-oxide (BOX) of $3 \mu \mathrm{m}$ (Fig. 2a), deep reactive ion etching (DRIE) was used to form $5 \mu \mathrm{m}$ wide trenches in the device layer, creating a pair of clampedclamped beams, as shown in the scanning electron micrograph (SEM) of Fig. 3. In addition, trenches were etched through the handle wafer, in order to define the device boundary and a block of the handle layer below the resonators (Fig. 2b). Hydrofluoric acid (HF) vapor was used to etch the BOX, resulting in the release of the resonators, the handle block below the resonators, and the device from the wafer grid (Fig. 2c).

The simple, rectangular clamped-clamped beam design was chosen for ease of fabrication, with comb drives for actuation and sensing disregarded in favor of a parallel plate design. In addition, rectangular beams enable straightforward theoretical calculations and finite-element-method (FEM) simulations to be performed to determine mode-localization effects.

The length of the beams has to be a minimum of $400 \mu \mathrm{m}$ in order to allow for a sufficient size of left and right electrode (Fig. 3), which are anchored areas and as such require appropriate dimensions to ensure sufficient BOX remains after the HF vapor etch. (a)

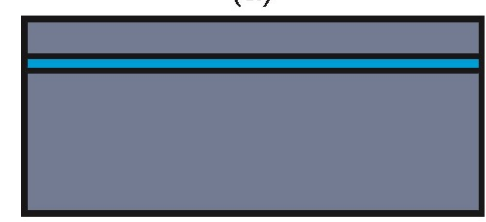

(b)

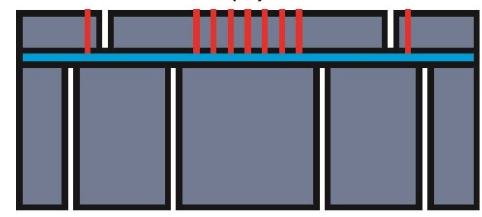

(c)

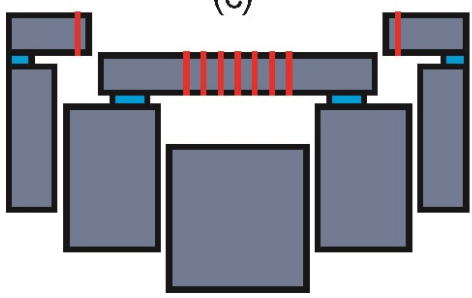

\section{$\square$ Silicon BOX $\square$ Release Holes}

Fig. 2. SOI fabrication process: (a) SOI wafer with $50 \mu \mathrm{m}$ device layer, $3 \mu \mathrm{m}$ BOX layer, and $560 \mu \mathrm{m}$ handle wafer (b) DRIE of front and back side trenches and release holes (c) HF vapor etch of BOX resulting in release of resonators and device from the wafer grid, with no stiction.

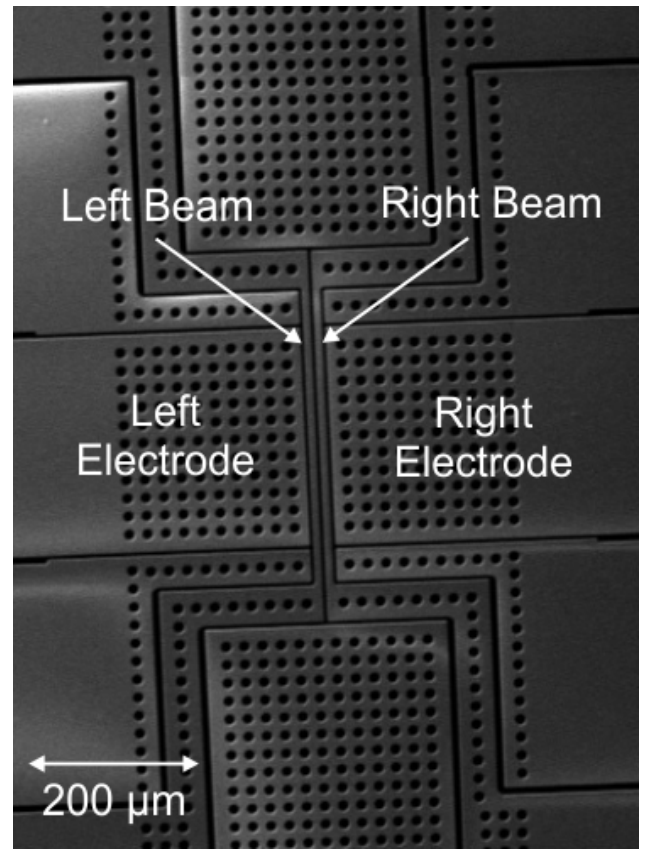

Fig. 3. Scanning electron micrograph (SEM) of coupled resonator device with $410 \times 10 \mu \mathrm{m}$ beams.

Two device designs were fabricated; one with beams of dimensions $410 \mu \mathrm{m} \times 10 \mu \mathrm{m}$ and the other $410 \mu \mathrm{m} \times$ $20 \mu \mathrm{m}$. The increase in width will result in an increase in the mechanical stiffness of the beams, allowing the effect on device sensitivity to be characterized. 

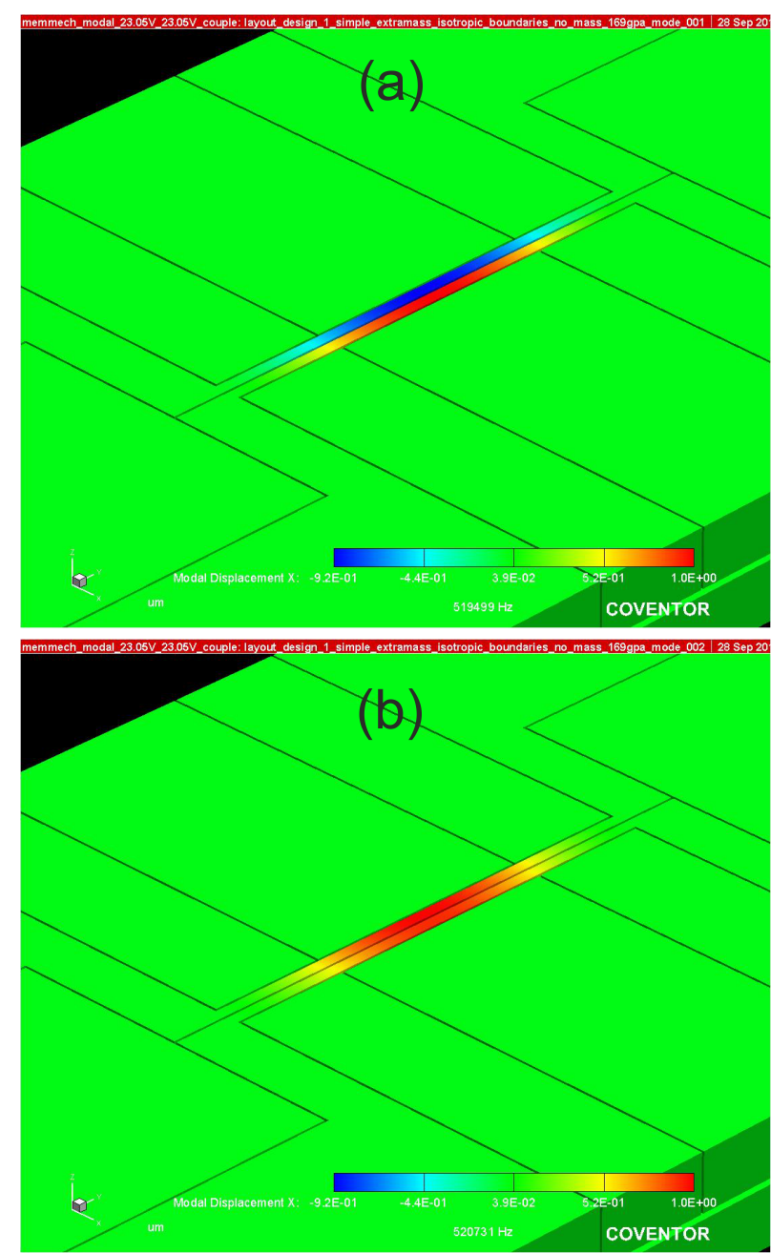

Fig. 4. CoventorWare FEM simulation 3D result of the (a) out-of-phase and (b) in-phase mode-shapes.

\section{FEM Simulation SET-UP}

CoventorWare was used to perform simulations to establish the mode-localization behavior of coupled-resonator devices in response to a stiffness perturbation.

Models of the fabricated devices were created with a mesh density of $20 \mu \mathrm{m} \times 20 \mu \mathrm{m}$ bricks, which provided the fastest computation time without sacrificing result accuracy. In addition, the loss to the surrounding substrate of the vibration energy was included in the simulation, as well as atmospheric damping.

An electrostatic coupling spring was formed between the two beams by applying $-120 \mathrm{~V}$ to the right beam and holding the left at $0 \mathrm{~V}$, creating a coupling spring between the beams of $-20.9 \mathrm{~N} / \mathrm{m}$, sufficient to avoid mode-aliasing. A perturbation was introduced to the system by reducing the DC voltage of the left electrode from a starting value of $120 \mathrm{~V}$, so that the stiffness of the left resonator is increased. Fig. 4a and Fig. 4b show the simulated out-of-phase and in-phase mode shapes, respectively, for a $410 \mu \mathrm{m} \times 10 \mu \mathrm{m}$ beam device.

The same simulation was performed for a model of a $410 \mu \mathrm{m} \times 20 \mu \mathrm{m}$ beam device. Focusing on the in-phase mode,

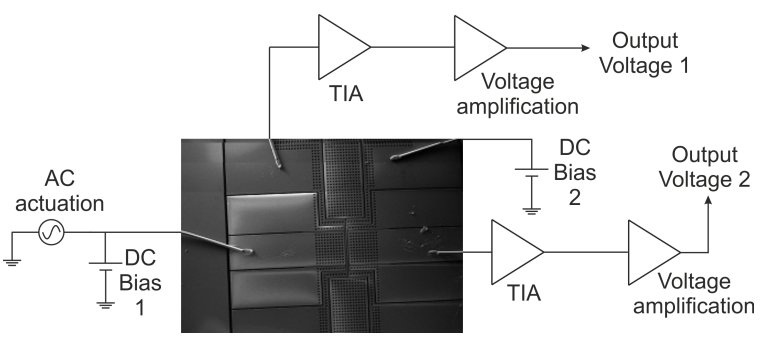

Fig. 5. Experimental set-up. MEMS device was wire-bonded to chip carrier positioned on printed circuit board containing output circuitry.

the response of the amplitude ratio of the two beams to a stiffness perturbation was extracted. The simulated response of the amplitude ratio will be shown alongside measurement results in section VI.

\section{EXPERIMENTAL SET-UP}

The testing set-up detailed in Fig. 5 was implemented in order to extract the amplitude ratio of the resonator pair. As with the simulation model, an electrostatic coupling spring of $-20.9 \mathrm{~N} / \mathrm{m}$ was formed between the two beams by applying $-120 \mathrm{~V}$ to the right beam and holding the left at $0 \mathrm{~V}$. A $1 \mathrm{~V}$ peak-to-peak AC signal was provided by a signal generator and was applied to the left electrode.

Transimpedence amplifiers (OPA657 from Texas Instruments) were used to convert the varying motional current from each resonator to a voltage. After further amplification, a spectrum analyzer (Rohde and Schwarz FSV 3) measured the amplitude of each of the two output voltages.

The MEMS device was wire bonded to a chip carrier, which was plugged into a socket on a printed circuit board (PCB) containing the measurement circuitry. The PCB was placed in a vacuum chamber at 0.02 mTorr, with suitable electrical feedthroughs.

The DC voltage of the left electrode was set at $120 \mathrm{~V}$ and the right electrode was held at $0 \mathrm{~V}$, so that the potential difference, $\Delta V$, between each resonator and its neighboring electrode was $120 \mathrm{~V}$, which electrostatically softened the stiffness of each resonator equally according to (4). Then, the DC voltage of the left electrode was reduced from the starting value of $120 \mathrm{~V}$, so that the stiffness of the left resonator increased, and the response of the amplitude ratio of the two output signals was extracted.

\section{Simulation / ExPERIMENTAL Results}

The same experiments were performed on the $10 \mu \mathrm{m}$ and $20 \mu \mathrm{m}$ wide beam devices. The stiffness of the left resonator was increased in increments so that the relative stiffness $(\Delta k / k)$ increases by up to $400 \mathrm{ppm}$. The initial in-phase mode frequency has been measured as $430 \mathrm{kHz}$ for the $10 \mu \mathrm{m}$ wide beam device and $955 \mathrm{kHz}$ for the $20 \mu \mathrm{m}$ wide beam device.

Fig. 6 shows the response of the amplitude ratio $\left(x_{1} / x_{2}\right)$ at the in-phase mode frequency to the shift in relative stiffness $(\Delta k / k)$ of the left resonator. It can be seen that the FEM simulated and experimental results show good agreement, for both the $10 \mu \mathrm{m}$ and $20 \mu \mathrm{m}$ wide beam devices. 


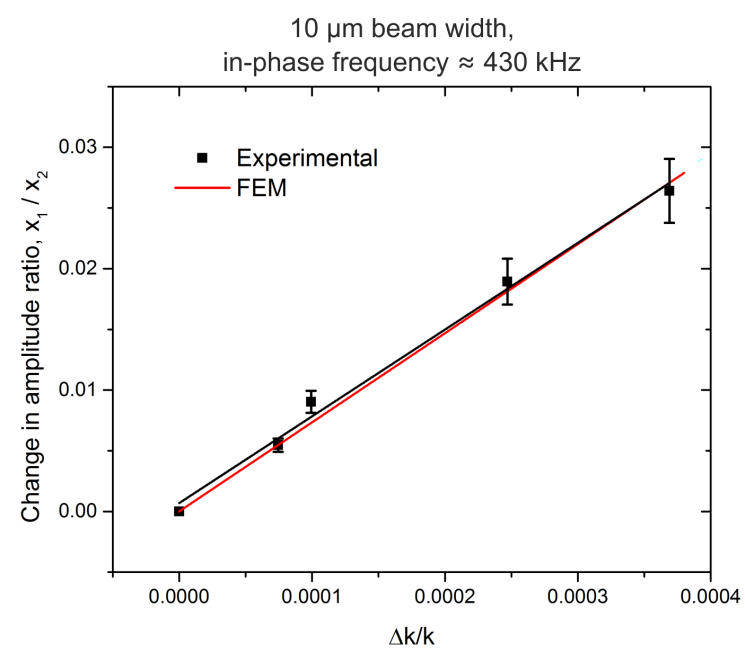

$20 \mu \mathrm{m}$ beam width,

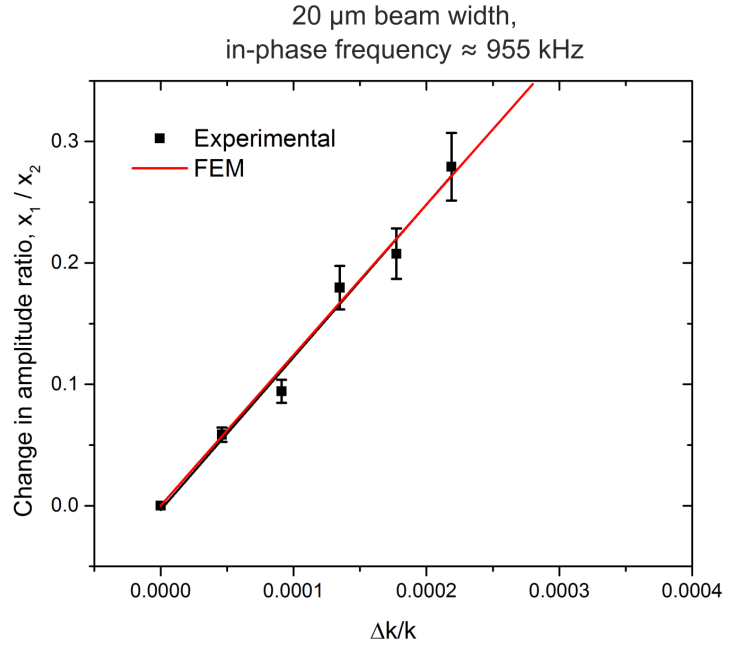

Fig. 6. Change in amplitude ratio at the in-phase mode frequency in response to stiffness change of left resonator.

The sensitivity, $S$, of the device is defined as the response of the amplitude ratio $\left(x_{1} / x_{2}\right)$ at the in-phase mode frequency to the shift in relative stiffness $(\Delta k / k)$ of the left resonator as given by

$$
S=\frac{\Delta\left(x_{1} / x_{2}\right)}{\Delta k / k}
$$

A sensitivity of 75 was measured for the $10 \mu \mathrm{m}$ wide beam and 1000 for the $20 \mu \mathrm{m}$ wide beam, which compares favorably to the current state-of-the-art value of 356 [3].

As discussed in section II, the sensitivity of a device to a relative change in the stiffness should be higher for devices with a higher initial stiffness. The results in Fig. 6 confirm the relationship with the sensitivity being 13 times higher for the $20 \mu \mathrm{m}$ wide beam device, compared with the $10 \mu \mathrm{m}$ wide beam device, because of its higher initial stiffness.

\section{CONCLUSION}

The mode-localization effect was utilized successfully to sense a stiffness change in one of a pair of electrostaticallycoupled MEMS clamped-clamped beams. It was shown that the amplitude ratio at the in-phase fundamental mode frequency shifts in response to a an electrostatically induced stiffness change in one of the resonators. Two devices with different beam widths were fabricated and tested, with a 13 times increase in sensitivity to relative stiffness change reported for devices with $20 \mu \mathrm{m}$ wide beams, compared with $10 \mu \mathrm{m}$ wide beams.

In addition, the clamped-clamped beams devices reported here exhibit a 2.8 times improvement in sensitivity compared to the state-of-the-art, with the added benefit of a simple device architecture and a high-yield, reliable SOI fabrication process.

\section{REFERENCES}

[1] A. A. Seshia, M. Palaniapan, T. A. Roessig, R. T. Howe, R. W. Gooch, T. R. Schimert, and S. Montague, "A Vacuum Packaged Surface Micromachined Resonant Accelerometer," J. Microelectromech. Syst., vol. 11, no. 6, pp. 784-793, Dec 2002.

[2] H. Zhang and E. S. Kim, "Micromachined Acoustic Resonant Mass Sensor," J. Microelectromech. Syst., vol. 14, no. 4, pp. 699-706, Aug. 2005.

[3] P. Thiruvenkatanathan, J. Yan, J. Woodhouse, and A. A. Seshia, "Enhancing Parametric Sensitivity in Electrically Coupled MEMS Resonators" J. Microelectromech. Syst., vol. 18, no. 5, pp. 1077-1086, Oct. 2009.

[4] I. Sari, I. Zeimpekis, and M. Kraft, "A dicing free SOI process for MEMS devices," Microelectron. Eng., vol. 95, pp. 121-129, Jul. 2012.

[5] P. Thiruvenkatanathan, J. Yan, and A. A. Seshia, "Differential Amplification of Structural Perturbations in Weakly Coupled MEMS Resonators" IEEE Trans. Ultrason. Ferroelectr. Freq. Control, vol. 57, no. 3, pp. 690-697, Mar. 2009. 